\title{
Mössbauer and heat capacity studies of $\mathrm{ErZnSn}_{2}$
}

Kazimierz Latka, Janusz Przewoźnik, Jan Zukrowski, Yuriy Verbovytskyy, Antonio Pereira Gonçalves

\begin{abstract}
Heat capacity results obtained for the intermetallic compound $\mathrm{ErZnSn}_{2}$ were re-analysed to also consider, apart from the classical Debye model, the anharmonicity of the crystal lattice and the proper set of Einstein modes. The ${ }^{119 \mathrm{~m}} \mathrm{Sn}$ Mössbauer technique was applied to derive the hyperfine interaction parameters characteristic of the two inequivalent crystallographic Sn sites in the compound studied. Quadrupole interaction constants, as measured by ${ }^{119 m} \mathrm{Sn}$ Mössbauer spectroscopy, allowed for estimations of $V_{z z}$ components of the electric field gradient tensor that exist at both Sn sites in the discussed compound.
\end{abstract}

Keywords: ternary rare-earth intermetallics $\bullet$ heat capacity $\bullet$ Mössbauer spectroscopy

K. Łątka

Marian Smoluchowski Institute of Physics,

Jagiellonian University,

11 Prof. S. Łojasiewicza Str., 30-348 Kraków, Poland,

Tel.: +48 12664 4668, Fax: +48 12664 4905,

E-mail: uflatka@cyf-kr.edu.pl

\section{J. Przewoźnik}

Department of Solid State Physics,

Faculty of Physics and Applied Computer Science,

AGH University of Science and Technology,

30 A. Mickiewicza Ave., 30-059 Kraków, Poland

\section{J. Żukrowski}

Department of Solid State Physics,

Faculty of Physics and Applied Computer Science,

AGH University of Science and Technology,

30 A. Mickiewicza Ave., 30-059 Kraków, Poland

and Academic Centre for Materials and Nanotechnology,

Faculty of Physics and Applied Computer Science,

AGH University of Science and Technology,

30 A. Mickiewicza Ave., 30-059 Kraków, Poland

Yu. Verbovytskyy

Department of Hydrogen Technologies and Hydride

Materials Science,

Karpenko Physico-Mechanical Institute

of the NAS of Ukraine,

5 Naukova Str., 79-060 Lviv, Ukraine

\section{A. P. Gonçalves}

C2TN, Campus Tecnológico e Nuclear,

Instituto Superior Técnico, Universidade de Lisboa,

10 Estrada Nacional Str., 2695-066, Bobadela LRS,

Portugal

Received: 30 June 2016

Accepted: 26 November 2016

\section{Introduction}

Recently, new series of ternary intermetallics of the chemical formula $\mathrm{RZnSn}_{2}$ (where $\mathrm{R}$ is substituted by the elements Y, Gd, Tb, Dy, Ho, Er, Tm, or Lu) were successfully synthesized. X-ray diffraction investigations showed that their crystalline structure is of a tetragonal $\mathrm{HfCuSi}_{2}$ type [1]. These compounds belong to the broad group of R(A)-T-X (where $\mathrm{R}$ stands for a rare-earth or A - actinide element, $\mathrm{T}$ is a $d$ metal, and $\mathrm{X}$ is a $p$ element) ternary intermetallics [2-21], which display numerous crystalline and magnetic structures and a wealth of intriguing physical properties like complex magnetic orderings, unconventional superconductivity or fluctuating valence. Magnetic susceptibility together with heat capacity results show that the compound $\mathrm{LuZnSn}_{2}$ does not undergo any magnetic phase transitions at temperatures as low as $1.9 \mathrm{~K}[1,22]$, and therefore this compound is an ideal reference material for the estimation of magnetic contributions to the heat capacity of magnetically ordered isostructural compounds, for example ErZnSn ${ }_{2}$ that shows a clear phase transition within the experimental temperature range between paramagnetic and antiferromagnetic states with a Néel temperature $T_{\mathrm{N}}=5.0 \mathrm{~K}$ [1]. Additionally, the antiferromagnetic character of the latter compound was corroborated by the observed negative value of its paramagnetic Curie temperature $\theta_{P}=-8.8 \mathrm{~K}$ as well as by the metamagnetic behaviour of the recorded magnetization curve measured at the lowest temperatures [1]. It is worth noting that the obtained effective magnetic moment, $\mu_{\text {eff }}=10.47 \cdot \mu_{\mathrm{B}}$, is remarkably higher than the expected theoretical value characteristic of the 
$\mathrm{Er}^{3+}$ free-ion value $\left(\mu_{\text {eff }}\right.$ (theor.) $=g \mu_{\mathrm{B}}[J(J+1)]^{1 / 2}=$ $\left.9.58 \mu_{\mathrm{B}}\right)$. The observed excess of effective magnetic moment has already been discussed [1], and might be interpreted as the contribution originating from the $d$-electrons of $\mathrm{Zn}$.

The main purpose of this report is to present and discuss a new analysis of heat capacity data [22] obtained for $\mathrm{ErZnSn}_{2}$ with the help of an extended Debye model that fits experimental data over the whole range of temperatures, in contrast to previous results [22] where a simple Debye model was used. Additionally, the results of a Mössbauer investigation conducted at room temperature with a ${ }^{119} \mathrm{Sn}$ source is reported and discussed with regard to the compound studied.

\section{Experimental}

The polycrystalline ErZnSn ${ }_{2}$ sample was prepared, as previously described [1], by melting the constituent elements at $\sim 1000^{\circ} \mathrm{C}$ followed by an annealing procedure at $400^{\circ} \mathrm{C}$. Its crystal structure was derived from an X-ray powder diffraction pattern by means of the Rietveld method using the FullProf program package [23] (see reference [1]).

Magnetic and heat capacity measurements were carried out for the bulk sample within the temperature range from $1.9 \mathrm{~K}$ to $300 \mathrm{~K}$ using a vibrating sample magnetometer (VSM) and the heat capacity (HC) insert of the Quantum Design Physical Property Measurement System (PPMS). The reported specific heat studies were performed in a zero external magnetic field. It must be stressed that great care was taken to properly correct the obtained raw HC data by accounting for the specific heat contribution originating from the supporting platform and the adhesive Apiezon $N$ addenda applied to couple our sample to the PPMS HC platform. For this purpose the HC curve of the supporting platform plus an appropriate amount of adhesive addenda (about $0.2 \mathrm{mg}$ ) was pre-measured and subtracted from the raw $\mathrm{HC}$ data.

The ${ }^{119} \mathrm{Sn}$ Mössbauer spectrum was measured in transmission geometry at room temperature by means of an electromechanical spectrometer of Kan-

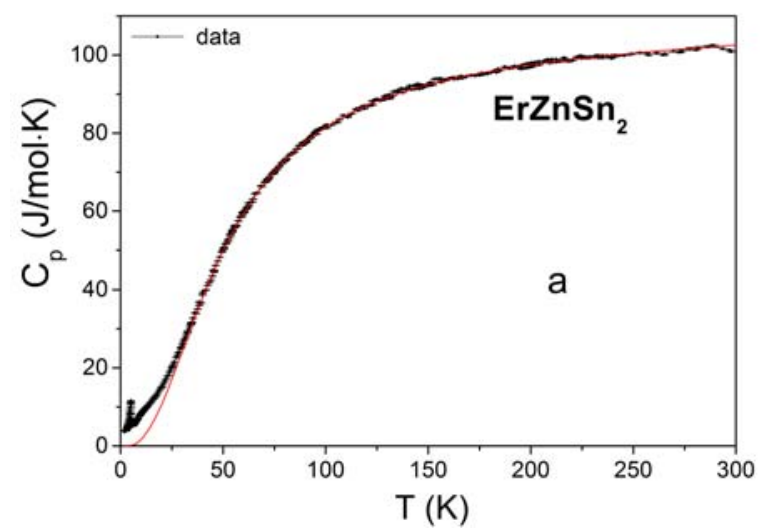

keleit type operating in the constant-acceleration mode utilizing a ${ }^{119 m}$ Sn source in the $\mathrm{CaSnO}_{3}$ matrix. A proportional counter was used to detect the incoming $23.875 \mathrm{keV} \gamma$-rays. The routine calibration of the velocity scale was conducted at room temperature using a standard ${ }^{57} \mathrm{Co}(\mathrm{Rh})$ source and a metallic iron foil. The obtained resonance absorption spectrum of ${ }^{119 \mathrm{~m}} \mathrm{Sn}$ was fitted by means of the least-squares fitting procedure within the transmission integral taking into account the full hyperfine Hamiltonian interaction for calculations of the resonance-line positions and their relative line intensities.

\section{Results and discussion}

\section{X-ray diffraction}

The analysis of the recorded X-ray diffractogram confirms that the ErZnSn ${ }_{2}$ compound crystallizes in a similar way to the tetragonal structure of $\mathrm{HfCuSi}_{2}(P 4 / \mathrm{nmm}$ space group). Two symmetrically inequivalent $\mathrm{Sn}$ sites, $2 a(\overrightarrow{4} \mathrm{~m} 2)$ and $2 c(4 \mathrm{~mm})$, exist in the unit cell and exhibit a $1: 1$ nominal occupational ratio [1].

\section{Heat capacity}

The measured specific heat capacity data are displayed in Figs. 1a and 1b. A sharp $\lambda$ type anomaly for $\mathrm{ErZnSn}_{2}$ was recorded at $T_{\mathrm{N}}=5.0 \mathrm{~K}$ in perfect agreement with the Néel temperature determined from the magnetic measurements taken under the influence of a low external magnetic field [1]. The magnetic contribution, $C_{p \text { magn }}$, for the compound $\mathrm{ErZnSn}_{2}$ can be separated from its total specific heat capacity, $C_{p}$, using the following equation:

$$
C_{p}=C_{\text {lat }}+C_{p \text { magn }}
$$

where $C_{\text {lat }}$ is the sum of the conduction electron $C_{e l}=\gamma T$ and the phonon part $C_{p h}$, i.e. the lattice contribution to the total specific heat capacity. Bearing in mind that $C_{p}(T)$ curves measured for Er and Lu intermetallics overlap in a good approximation at

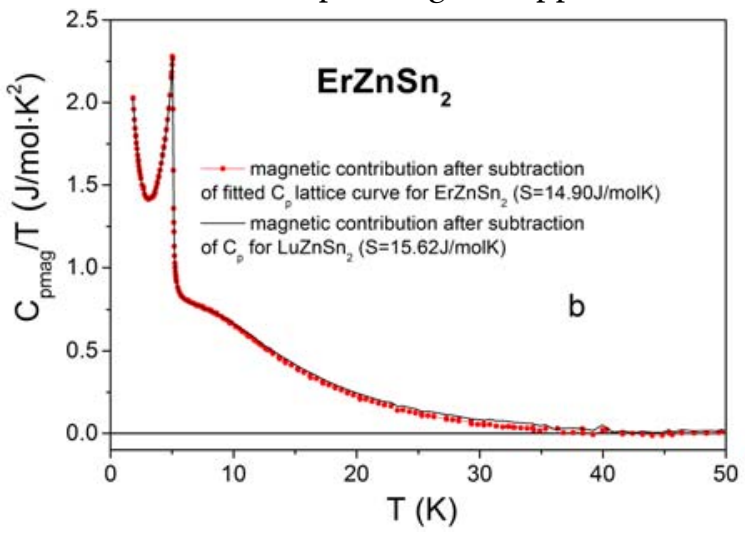

Fig. 1. Temperature dependencies of the specific heat capacity $\left(C_{p}\right)$ for $\mathrm{ErZnSn}_{2}$ together with fitted $C_{p}$ lattice contributions (as explained in the text) (a) and $C_{p \text { magn }} / T$ (b) where $C_{p \text { magn }}$ was obtained by subtracting the fitted $C_{p}$ lattice curve (i.e. the curve in red, Fig. 1a) or the $C_{p}$ for the non-magnetic reference material, i.e. $\mathrm{LuZnSn}_{2}$ (continuous line in black, Fig. 1b). The derived fitting parameters are gathered in Table 1. 
Table 1. The results for the derived fitting parameters from specific heat capacity data obtained for $\mathrm{ErZnSn}_{2}$ using Eq. (1)

\begin{tabular}{lccc}
\hline & Value & Multiplicity \\
$m_{\mathrm{i}}$ & Remarks \\
\hline$\theta_{\mathrm{D}}$ & $(141.78 \pm 0.34) \mathrm{K}$ & - & settled as for $\mathrm{LuZnSn}_{2}[24]$ \\
$\theta_{\mathrm{E} 1}$ & $(56.38 \pm 0.11) \mathrm{K}$ & $\times 1$ & settled as for $\mathrm{LuZnSn}_{2}[24]$ \\
$\theta_{\mathrm{E} 2}$ & $(148.31 \pm 0.71) \mathrm{K}$ & $\times 5$ & fitted \\
$\theta_{\mathrm{E} 3}$ & $(262.30 \pm 1.60) \mathrm{K}$ & $\times 3$ & fitted \\
$\alpha$ & $(12.79 \pm 0.26) \times 10^{-5} \mathrm{~K}^{-1}$ & - & fitted \\
$\gamma$ & $(5.28 \pm 0.05) \times 10^{-3} \mathrm{~J} \cdot \mathrm{mol}^{-1} \cdot \mathrm{K}^{-2}$ & - & settled as for $\mathrm{LuZnSn}_{2}[24]$ \\
\hline
\end{tabular}

temperatures above $\sim 50 \mathrm{~K}[22]$, one can choose the compound $\mathrm{LuZnSn}_{2}$ as a good reference material making the rough estimation of $C_{\text {lat }}$ for $\mathrm{ErZnSn}_{2}$ and for other magnetic members of the $\mathrm{RZnSn}_{2}$ series possible. Therefore, it is obvious that by subtracting both curves the $C_{p \operatorname{magn}}(T)$ value for the Er compound can be calculated. In turn, integration of $C_{p \operatorname{magn}}(T) / T$ yields the magnetic entropy contribution, $S_{\text {magn, }}$ amounts to $15.62 \mathrm{~J} /(\mathrm{mol} \cdot \mathrm{K}$ ) (or $14.90 \mathrm{~J} /(\mathrm{mol} \cdot \mathrm{K}$ ) when the fitted curve in red is used) as shown in Fig. $1 \mathrm{~b}$, which is less than the value $R \ln 16=23.05$ $\mathrm{J} /(\mathrm{mol} \cdot \mathrm{K})$ expected for the ground state $J=15 / 2$ multiplet of $\mathrm{Er}^{3+}$.

It is worth mentioning that the simple formula for $C_{\text {lat }}$

$$
C_{l a t}=C_{e l}+C_{p h}=\gamma T+C_{p h}
$$

where $C_{p h}$ is described by the classical Debye formula:

$$
C_{p h}=9 R\left(\frac{T}{\theta_{\mathrm{D}}}\right)^{3} \int_{0}^{\frac{\theta_{\mathrm{D}}}{T}} \frac{x^{4} e^{x}}{\left(e^{x}-1\right)^{2}} d x
$$

is not suitable for the fitting of $C_{p}(T)$ dependencies measured for the intermetallics $\mathrm{Y}$ and $\mathrm{Lu}$ including that for Er far above $T_{\mathrm{N}}$ [22]. However, it was shown that the experimental heat capacity data, $C_{\text {lat }}(T)$, for $\mathrm{LuZnSn}_{2}$ [24] and $\mathrm{ErZnSn}_{2}$, as expounded in this work, can be successfully fitted over the whole temperature range by taking into account anharmonic effects that occur at elevated temperatures, as well as by the inclusion of selected Einstein modes for $\mathrm{LuZnSn}_{2}$ and $\mathrm{ErZnSn}_{2}$ respectively using the extended Debye equation in the form:

$$
\begin{aligned}
C_{p} & =C_{p h+e l}=9 R \frac{1}{1-\alpha T}\left(\frac{T}{\theta_{\mathrm{D}}}\right)^{3} \int_{0}^{\frac{\theta_{\mathrm{D}}}{T}} \frac{x^{4} e^{x}}{\left(e^{x}-1\right)^{2}} d x \\
& +R \frac{1}{1-\alpha T} \sum_{i} m_{i} \frac{\left(\frac{\theta_{\mathrm{E}_{i}}}{T}\right)^{2} e^{\theta_{\mathrm{E}_{i}} / T}}{\left(e^{\theta_{\mathrm{E}_{i}} / T}-1\right)^{2}}+\gamma T
\end{aligned}
$$

Here $\theta_{\mathrm{D}}$ is the Debye temperature, $\theta_{\mathrm{E} i}(i=1,2$ or 3 ) are the Einstein temperatures, $\alpha$ stands for the anharmonic coefficient, $R$ denotes the gas constant and $\gamma$ is an electronic specific heat coefficient. There are 3 acoustic and 9 optical modes, which are described by the first and second terms of Eq. (4), respectively. The obtained values for all these parameters from the above-mentioned fit procedure are displayed in
Table 1 together with the applied multiplicities $m_{i}$ for each individual Einstein branch $\mathrm{E}_{i}$.

\section{${ }^{119}$ Sn Mössbauer spectroscopy}

The point symmetries of Sn environments, i.e. $\overline{4} m 2$ ( $2 a$ site) and $4 m m$ ( $2 c$ site), ensure non-zero values of the axially symmetric (with asymmetry parameter $\eta=0$ ) electric field gradient (EFG) tensor at these sites, and as a consequence, non-zero electric quadrupole hyperfine interaction parameters at both $\mathrm{Sn}$ positions in the $\mathrm{ErZnSn}_{2}$ lattice. In accordance with the two non-cubic environments of Sn atoms in the crystal structure, i.e. $2 a$ and $2 c$ sites, the spectrum recorded at room temperature can be reasonably fitted with two quadrupole-split components including a single line of the metallic impurity tin (with isomer shift $\delta_{\text {IS }}$ kept constant and equal to $2.552(9) \mathrm{mm} / \mathrm{s}$ [25]), which is also evident from X-ray diffraction analysis and originates from the synthesis procedure as well as the known decomposition process of the probe over time. Similarly to the case of $\mathrm{LuZnSn}_{2}$ [24], the resonance spectrum recorded for $\mathrm{ErZnSn}_{2}$ at room temperature can be fitted with different pairs of main quadrupole split subsets of hyperfine parameters (together with the respective pairs of isomer shifts) for two crystallographically inequivalent Sn sites. However, from a physical point of view, a fit with only two different quadrupole interaction constants and comparable isomer shifts for both Sn sites (Fig. 2) is acceptable. One can underline here that such a situation is observed in the closely

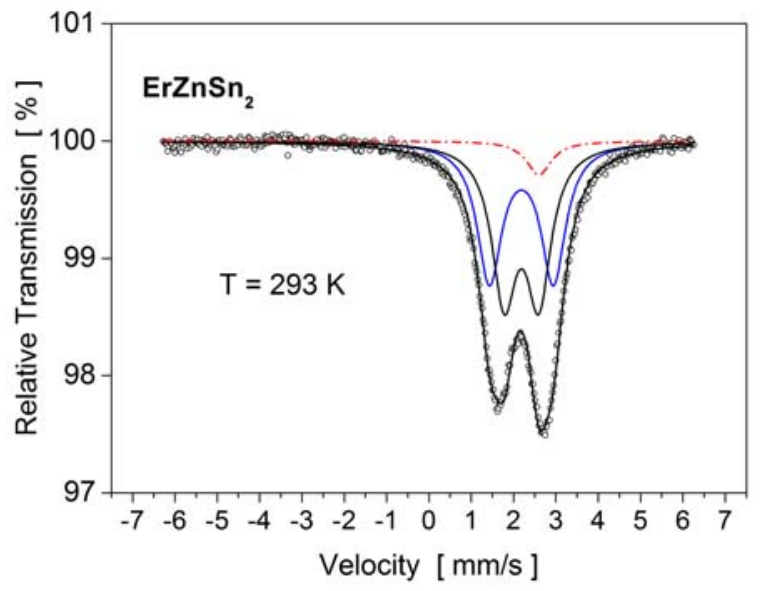

Fig. 2. ${ }^{119} \mathrm{Sn}$ Mössbauer spectrum of $\mathrm{ErZnSn}_{2}$ at room temperature fitted with two quadrupole doublets and one strictly settled single line metallic tin impurity component (see text for explanation). 
Table 2. Hyperfine interaction parameters inferred from the ${ }^{119} \mathrm{Sn}$ resonance spectrum obtained for the intermetallic compound $\mathrm{ErZnSn}_{2}$ at $293 \mathrm{~K}$

\begin{tabular}{lcccc}
\hline & Component & $\begin{array}{c}\delta_{\mathrm{IS}} \\
{[\mathrm{mm} / \mathrm{s}]}\end{array}$ & $\begin{array}{c}\left|\Delta E_{Q}\right| * \\
{[\mathrm{~mm} / \mathrm{s}]}\end{array}$ & $\begin{array}{c}\text { Relative area } \\
{[\%]}\end{array}$ \\
\hline I & $2.185(3)$ & $3.019(24)$ & $45.2(1.2)$ & $\chi^{2}$ \\
II & $2.186(3)$ & $1.634(20)$ & $49.3(1.3)$ & 1.1575 \\
\hline$\Delta E_{Q}=e Q V_{z z}=2 \Delta$ where $\Delta$ is the overall quadrupole splitting for a given component in the recorded spectrum (see Fig. 2).
\end{tabular}

related series of defected $\mathrm{GdT}_{x} \mathrm{Sn}_{2}$ compounds [21], where Sn atoms also occupy two different symmetry positions and where Mössbauer spectra can be fitted in a suitable way with only two different quadrupole splittings, but with very similar isomer shifts for both $\mathrm{Sn}$ sites. On the other hand, an assumption that isomer shifts should be significantly different at Sn sites seems to also be unreasonable, since for $\mathrm{Sn}$ atoms their transformation requires a rather big change in the valence electron $s p$ configuration of their own tin atoms [24], and this is not expected here, as in the $2 a$ position Sn atoms interact directly with the other four Sn and Er atoms, respectively, while at the $2 c$ site $\mathrm{Sn}$ atoms interact with four $\mathrm{Zn}$ (instead of Sn) and Er atoms, respectively, but valence electron $s$ configurations of Sn and $\mathrm{Zn}$ atoms remain roughly identical. The observed systematics of the isomer shifts of $\mathrm{Sn}$ in most intermetallic systems (including ternary systems) [26] is in line with the previous statement, showing that the effective number of $5 s$ tin electrons is merely the same and not much different than one (see Table 9a in [26]). In this context, one can state that a theoretical support in predicting Mössbauer parameters would be of great importance. In particular, the theoretical calculations could enable the right association of the obtained sets of experimental results for hyperfine parameters for a given site in the crystallographic structure and also facilitate the correct interpretation of the derived isomer shifts and quadrupole splittings, giving a deeper insight into the electronic structure of the studied material. In this regard one can only speculate, for example, that a larger quadrupole splitting could be associated with a lower symmetry site of Sn. Nowadays, the application of density functional theory (DFT) is quite successful in this regard, but to the best of our knowledge such calculations were not made for Sn hyperfine parameters in the system studied.

Generally, to obtain reliable results from a given fitting procedure, some parameters, like half-widths for source $\Gamma_{s}$ and absorber $\Gamma_{a}$, have to be constrained and kept constant, being equal to those derived from earlier experiments with reference to the absorber $\mathrm{BaSnO}_{3}$. In this case these values are as follows: $\Gamma_{s}=0.37 \mathrm{~mm} / \mathrm{s}$ and $\Gamma_{a}=0.33 \mathrm{~mm} / \mathrm{s}$. Additionally, only fits with comparable relative intensities for both quadrupole components were acceptable to reflect the fact that the occupation of the above-mentioned Sn sites is equal. It must be stressed, that fits with constrained amplitudes of main Sn contributions with their $1: 1$ ratio, do not remarkably change the qoodnes, $\chi^{2}$, of such a fit. Small differences between relative intensities can always be explained by possible differences in Debye-Waller $f$ factors for both symmetry sites of tin. This is the main reason why in Table 2 the derived Mössbauer results are presented for fits with unconstrained main Sn contributions. The subtraction of the sum of the derived intensities for both quadrupole components from $100 \%$ yields the contribution of the Sn impurity being equal to about 5.5\% (see Table 2).

The required values of the electric field gradient $V_{z z}$ at the tin nuclei can be obtained using the quadrupole interaction constants $\Delta E_{Q}=e Q V_{z z}$ (where the nuclear quadrupole moment $Q$ for the first excited state of ${ }^{119 m} \mathrm{Sn}$ is equal to $-0.094 \mathrm{~b}$ [27]) given in Table 2 by the simple relation: $V_{z z}=-8.47128 \times$ $10^{21} \Delta E_{Q}[\mathrm{~mm} / \mathrm{s}] \mathrm{V} / \mathrm{m}^{2}$ and they are $\left|V_{z z 1}\right|=25.6 \times$ $10^{21} \mathrm{~V} / \mathrm{m}^{2}$ and $\left|V_{z z 2}\right|=13.8 \times 10^{21} \mathrm{~V} / \mathrm{m}^{2}$, respectively.

\section{Conclusions}

It was shown here that the experimental specific heat capacity data, $C_{p}(T)$, for $\mathrm{ErZnSn}_{2}$ can be successfully fitted over the whole temperature range using an extended Debye model which takes into account the effect of lattice anharmonicity and the suitable number of Einstein modes.

Mössbauer spectra can be fitted in a suitable way with only two different quadrupole splittings, but with almost the same isomer shifts for both Sn sites.

Those obtained by ${ }^{119 m} \mathrm{Sn}$ Mössbauer spectroscopy quadrupole interaction constants enabled the determination of very important $V_{z z}$ components of electric field gradient (EFG) tensors at both Sn sites of $\mathrm{ErZnSn}_{2}$ in the field of solid-state physics. The derived isomer shifts of about $2.19 \mathrm{~mm} / \mathrm{s}$ are characteristic of tin in metallic systems.

Acknowledgments. The magnetic and specific heat capacity measurements were performed by the Quantum Design PPMS system as well as a Mössbauer spectroscopy facility funded by the financial support of the European Regional Development Fund in the framework of the Polish Innovative Economy Operational Programme (contract no. POIG.02.01.00-12-023/08).

\section{References}

1. Verbovytskyy, Yu., Łątka, K., Przewoźnik, J., Leal, N., \& Gonçalves, A. P. (2012). On the new ternary $\mathrm{RZnSn}_{2}$ compounds with $\mathrm{HfCuSi}_{2}$ structure type. Intermetallics, 20, 176-182. DOI: 10.1016/j.intermet.2011.08.024.

2. Görlich, E. A., Kmieć, R., Łątka, K., Matlak, T., Ruebenbauer, K., Szytuła, A., \& Tomala, K. (1975). Transferred hyperfine fields at the tin site in the Heu- 
sler-type alloys $\mathrm{Co}_{2} \mathrm{YSn}(\mathrm{Y}=\mathrm{Ti}, \mathrm{Zr}, \mathrm{Hf}, \mathrm{V})$. Phys. Status Solidi A-Appl. Mat., 30, 765-770. DOI: 10.1002/ pssa.2210300237.

3. Görlich, E. A., Kmieć, R., Łatka, K., Szytuła, A., \& Zygmunt, A. (1994). Magnetic properties and ${ }^{119} \mathrm{Sn}$ hyperfine interactions investigated in $\mathrm{RCoSn}(\mathrm{R}=\mathrm{Tb}$, Dy, Ho, Er) compounds. J. Phys.-Condens. Matter, 6 , 11127-11139. DOI: 10.1088/0953-8984/6/50/020.

4. Troć, R., Tran, V. H., Kolenda, M., Kruk, R., Łątka, K., Szytuła, A., Rossat-Mignod, J., Bonnet, M., \& Büchner, B. (1995). X-ray and neutron diffraction studies of UPdSn. J. Magn. Magn. Mater., 151, 102-110. DOI: 10.1016/0304-8853(95)00396-7.

5. Kruk, R., Kmieć, R., Łątka, K., Tomala, K., Troć, R., \& Tran, V. H. (1996). ${ }^{119}$ Sn Mössbauer studies of the compound UAuSn. J. Alloy. Compd., 232, L8-L11. DOI: 10.1016/0925-8388(95)02008-X.

6. Kruk, R., Kmieć, R., Łątka, K., Tomala, K., Troć, R., \& Tran, V. H. (1997). Magnetic properties of UTSn compounds ( $\mathrm{T}=\mathrm{Co}$, Rh, Ir, Ru) studied by ${ }^{119} \mathrm{Sn}$ Mössbauer spectroscopy. Phys. Rev. B, 55, 5851-5857. DOI: 10.1103/PhysRevB.55.5851

7. Łatka, K., Görlich, E. A., Chajec, W., Kmieć, R., \& Pacyna, A. W. (1997). Crystal and magnetic properties of GdTSn compounds (T = Ag, Au).J. Alloy. Compd., 262/263, 108-113.

8. Łątka, K., Görlich, E. A., Kmieć, R., Kruk, R., Pacyna, A. W., \& Chajec, W. (1998). Looking at unusual magnetism in rare-earth intermetallics with ${ }^{119} \mathrm{Sn}$ Mössbauer spectroscopy. Mol. Phys. Rep., 22, 87-97.

9. Görlich, E. A., Łatka, K., Kmieć, R., \& Warkocki, W. (1998). Hyperfine interactions of ${ }^{155} \mathrm{Gd}$ in the spinglass systems GdAgSn and GdAuSn. Mol. Phys. Rep., $22,35-40$.

10. Łątka, K., Kmieć, R., \& Gurgul, J. (2001). ${ }^{119}$ Sn Mössbauer spectroscopy studies of RAgSn compounds (R=La, Ce, Pr). J. Alloy. Compd., 319, 43-49. DOI: 10.1016/S0925-8388(01)00896-9.

11. Łątka, K., Chajec, W., Kmieć, R., \& Pacyna, A. W. J. (2001). Magnetic susceptibility and ${ }^{119}$ Sn Mössbauer spectroscopy studies of RAuSn compounds $(\mathrm{R}=\mathrm{La}$, Ce, Pr). J. Magn. Magn. Mater., 224, 241-248. DOI: 10.1016/S0304-8853(01)00035-X

12. Łątka, K., Kmieć, R., Kruk, R., Pacyna, A. W., Rams, M., Schmidt, T., \& Pöttgen, R. (2003). Exotic phase transitions in RERhSn compounds. Nukleonika, 48(S1), S35-S40.

13. Łatka, K., Kmieć, R., Kruk, R., Pacyna, A. W., Rams, M., Schmidt, T., Kotzyba, G., Pöttgen, R., \& Johrendt, D. (2003). Structure and properties of CeRhSn - a valence fluctuating system. Acta Phys. Pol. B, 34(2), $1225-1229$.

14. Łątka, K., Kmieć, R., Gurgul, J., Rams, M., Pacyna, A. W., Schmidt, T., \& Pöttgen, R. (2005). Structure, magnetic properties and ${ }^{119}$ Sn Mössbauer spectroscopy of PrRhSn. J. Solid State Chem., 178, 3101-3109. DOI: 10.1016/j.jssc.2005.06.041.
15. Schmidt, T., Johrendt, D., Sebastian, C. P., Pöttgen, R., Łatka, K., \& Kmieć, R. (2005). Structure, chemical bonding and ${ }^{119} \mathrm{Sn}$ Mössbauer spectroscopy of LaRhSn and CeRhSn. Z. Naturforsch., 60b, 1036-1042.

16. Łątka, K., Kmieć, R., Kruk, R., Pacyna, A. W., Fickenscher, T., Hoffmann, R. -D., \& Pöttgen, R. (2005). Structure, magnetic properties and Mössbauer spectroscopy of GdRhSn. J. Solid State Chem., 178, 2077-2090. DOI: 10.1016/j.jssc.2005.04.010.

17. Łątka, K., Kmieć, R., Gurgul, J., Pacyna, A. W., Rams, M., Schmidt, T., \& Pöttgen, R. (2006). Magnetic ordering in NdRhSn. J. Magn. Magn. Mater., 301, 359-370. DOI: 10.1016/j.jmmm.2005.07.009.

18. Łątka, K., Kmieć, R., Kruk, R., Pacyna, A. W., Rams, M., Schmidt, T., \& Pöttgen, R. (2008). Electronic and magnetic properties of ternary stannides RERhSn $(\mathrm{RE}=$ light rare-earth metals). J. Magn. Magn. Mater., 320(3/4), L18-L20. DOI: 10.1016/j.jmmm.2007.05.031.

19. Łatka, K., Pacyna, A. W., Pöttgen, R., \& Schappacher, F. M. (2008). Puzzling magnetism of $\mathrm{Gd}_{3} \mathrm{Cu}_{4} \mathrm{Sn}_{4}$. Acta Phys. Pol. A, 114, 1501-1508.

20. Łątka, K., Kruk, R., Kmieć, R., Troć, R., \& Tran, V. H. (2010). ${ }^{119}$ Sn Mössbauer spectroscopy in the heavy-fermion ferrimagnet $\mathrm{UCu}_{5}$ Sn". J. Phys.-Conf. Series, 217, 012136(4pp.). DOI: 10.1088/17426596/217/1/012136.

21. Görlich, E. A., Łatka, K., Kmieć, R., Tomkowicz, Z., \& Warkocki, W. (2000). Hyperfine interactions of ${ }^{119} \mathrm{Sn}$ and ${ }^{155} \mathrm{Gd}$ versus bulk properties of $\mathrm{GdT}_{x} \mathrm{Sn}_{2}$ phases. Mol. Phys. Rep., 30, 59-65.

22. Łątka, K., Przewoźnik, J., Verbovytskyy, Yu., \& Gonçalves, A. P. (2013). Thermodynamic properties of $\mathrm{RZnSn}_{2}(\mathrm{R}=\mathrm{Y}, \mathrm{Er}, \mathrm{Lu})$ compounds with $\mathrm{HfCuSi}_{2}$ structure type. Solid State Phenom., 194, 67-70. DOI: 10.4028/www.scientific.net/SSP.194.67.

23. Rodriguez-Carvajal, J., \& Roisnel, T. (1998). FullProf.98 and WinPLOTR: New Windows 95/NT Applications for Diffraction Commission for Powder Diffraction. International Union for Crystallography Newsletter, 20 (May-August) Summer.

24. Łątka, K., Przewoźnik, J., Żukrowski, J., Verbovytskyy, Yu., \& Gonçalves, A. P. (2015). Analysis of heat capacity and Mössbauer data for $\mathrm{LuZnSn}_{2} \mathrm{com}-$ pound. Nukleonika, 60(1), 97-101. DOI: 10.1515/ nuka-2015-0020.

25. Stevens, J. G., \& Gettys, W. I. (1978). Mössbauer isomer shift reference scales. In G. K. Shenoy, \& F. E. Wagner (Eds.), Mössbauer isomer shifts (pp. 901-906). Amsterdam: North-Holland Publishing Co.

26. Flinn, P. (1978). Tin isomer shifts. In G. K. Shenoy, \& F. E. Wagner (Eds.), Mössbauer isomer shifts (pp 593-616). Amsterdam: North-Holland Publishing Co.

27. Mössbauer Effect Data Center (MEDC). (2010, July). Properties of isotopes relevant to Mössbauer spectroscopy. ${ }^{119} \mathrm{Sn}$ isotope properties. Retrieved June, 3, 2014, from http://www.medc.dicp.ac.cn/Resourcesisotopes/Resource-Sn.php. 